\title{
Present and Future Detection of Terrestrial Biomarkers on Earthshine
}

\author{
Danielle Briot $^{1}$, Luc Arnold ${ }^{2}$, Stéphane Jacquemoud ${ }^{3}$ \\ and Jean Schneider ${ }^{4}$
}

\author{
${ }^{1}$ GEPI, Observatoire de Paris, 61 avenue de l'Observatoire, 75014 Paris, France \\ email: danielle.briot@obspm.fr \\ ${ }^{2}$ Observatoire de Haute-Provence, 04870 Saint-Michel l'Observatoire, France \\ email: luc.arnold@oamp.fr \\ ${ }^{3}$ Université Paris Diderot/ Institut de Physique du Globe de Paris \\ 35 rue Hélène Brion, 75013 Paris, France \\ email: jacquemoud@ipgp.fr \\ ${ }^{4}$ LUTH, Observatoire de Paris, CNRS, Universit Paris Diderot, \\ 5 place Jules Janssen, 92195 Meudon, France \\ email: jean.schneider@obspm.fr
}

\begin{abstract}
In the context of life detection on terrestrial exoplanets, new methods of search for spectral signatures of chlorophyll and other biomarkers in the Earthshine have been developed in the last few decades. Astronomical observations made at OHP and ESO (NTT) showed a significant signal when continents are facing the Moon. This signal, called the Vegetation Red Edge (VRE), is undoubtedly due to chlorophyll absorption properties. In order to strengthen these results, the LUCAS (LUmière Cendrée en Antarctique par Spectroscopie) project dedicated to the measurement of the Earthshine from the Concordia Research Station (C Dome, Antarctica) has been set up. One of the objectives of LUCAS was to observe prolonged variations of the VRE corresponding to various parts of the Earth facing the Moon. An extension of this project, called LUCAS II, would allow long-term observations to detect seasonal variations of the vegetation signal. These data, together with accurate measurements of the Earth's albedo, will help validate a model of global and spectral albedo of our planet.
\end{abstract}

Keywords. Astrobiology, Extraterrestrial life, Earth, Moon, Earthshine, Vegetation

\section{Introduction}

To anticipate the analysis of terrestrial exoplanet images, in particular the detection of life, we investigate the Earthshine that can be linked with the global Earth's albedo, as showed by Arnold et al. (2002) and Woolf et al. (2002). If life exists on an extrasolar planet, it may present unusual and unknown forms. In the absence of information, we look for proofs similar to those that prevail on Earth. We can explore classical biosignatures like $\mathrm{O}_{2}, \mathrm{O}_{3}, \mathrm{CH}_{4}, \mathrm{H}_{2} \mathrm{O}$ and $\mathrm{CO}_{2}$ absorption features, but also the Vegetation Red Edge (VRE) that is due to the presence of chlorophyllian pigments in plant leaves. As early as 1912, Arcichovsky suggested to look for chlorophyll absorption in the Earthshine spectrum, with the aim of calibrating chlorophyll in the spectrum of other planets (Arcichovsky 1912), but at that time, the spectral resolution of Earthshine observations was inadequate for that purpose (Tikhoff 1914, Danjon 1928). This idea was completely forgotten until about 1998, when Jean Schneider proposed to do such observations again. Following a first attempt made at the Observatoire de Haute Provence (Arnold et al. 2002), which successfully showed the feasibility of such a measurement, we used the New Technology Telescope of ESO to measure the VRE. We obtained a 
lower signal $(\mathrm{VRE}=1.3 \%)$ when the Pacific Ocean faces the Moon than when Africa faces the Moon (VRE $=3-4 \%$ ) (Hamdani et al. 2006). These observations also showed a significant variation of the Earth's albedo due to the cloud cover, implying that "pale blue dot" is rather white. A wide range of VRE values have been obtained from 2001 to 2006, as seen in the review paper of Arnold (2008).

\section{Interest of observations from Antarctic, Dome C}

The Earthshine can be observed in intermediate and low latitude during a few days before and after the New Moon, at every lunar cycle. It requires twilight observations, in the evening of the first days of the cycle (waxing Moon) and in the morning of the last days (waning Moon). In both case, such observations are limited in time. The disk of the Earth that faces the Moon depends on the location of the telescope and on the lighted part of the Earth, i.e. in the West of the telescope in the evening and in the East in the morning. Schneider (2002, private communication) suggested that the Earthshine could be observed during several hours, if observations were made from a site located in high latitude. Near the pole, it can even last 24 hours (total nycthemeron). During such a long observing window, different "landscapes", continents and oceans, alternately face the Moon because of the Earth's rotation. Antarctica is an ideal site for that kind of measurements. The Concordia research station, which is a joint French-Italian base dedicated to scientific research, is located at Dome C, one of the highest points of the Antarctic Plateau (latitude: $-75^{\circ} 06^{\prime}$ South, longitude: $123^{\circ} 23^{\prime}$ East, altitude: $3250 \mathrm{~m}$ ). The mean air temperature is $-50.8^{\circ} \mathrm{C}$, and the lowest value $-84.4^{\circ} \mathrm{C}$. The polar night lasts three months. Since 2005, when the station first opened to winter-over teams, it demonstrated exceptional conditions for astronomical observations, especially in the infrared (Fossat 2011). At this latitude, the Earthshine can be viewed about 8 times a year. From the March equinox to the June solstice, observations are possible during the last days of the lunar cycle and, from the June solstice up to the September equinox, they are possible during the first days of the lunar cycle. Preliminary experiments carried out the first winter confirmed that Earthshine's observations were possible during the twilight.

\section{LUCAS program (LUmière Cendrée en Antarctique par Spectroscopie)}

When Earthshine observations appeared to be possible from Concordia, we decided to measure the Vegetation Red Edge and other biomarkers by setting up the LUCAS (LUmière Cendrée en Antarctique par Spectroscopie) experiment. We took the opportunity of the first observational winter season to detect, analyze, and correct the instrumental problems that unfortunately occurred due to extreme temperatures. The year after, we made improvements that allowed us to obtain Moon spectra during all the observational sequences, from the winter (June) solstice to the end of observing time. Observations of the Earthshine could be carried out continuously during 6 hours, which is impossible in moderate or low latitude.

\section{More observations of Earthshine from Antarctica : LUCAS II}

It would be very interesting to perform longer Earthshine observations from Antarctica to determine the variations of the Earth's global albedo during the year, and to detect climate anomalies in the context of global change. Results could be completed by in situ and satellite observations. Experiments made in the northern high latitudes would 
allow to double the number of Earthshine observations. They would occur as long as in southern high latitudes, but in the opposite configuration. The LUCAS II program will reuse part of the LUCAS' instrumentation at the Concordia station. By inverting the PROSAIL radiative transfer model, we expect to detect seasonal variations of the vegetation signal as a function of the Leaf Area Index (LAI). PROSAIL is the short name of two coupled models that are very popular in the remote sensing community: PROSPECT, a leaf optical properties model (Jacquemoud \& Baret 1990), and SAIL, a canopy reflectance model (Verhoef 1984). It could be possible to estimate the average amount of vegetation present on Earth. The interaction with the atmosphere can be taken into account using a radiative transfer model through the atmosphere.

\section{Conclusion}

Long time observations of Earthshine and its seasonal variations would help to prepare the future detection of seasonal variations on extrasolar Earth-like planets.

Concerning observations of Earthshine, some geophysical applications also exist, according to the recommendations made by the NASA Navigator Program: "Continued observations of Earthshine are needed to discern diurnal, seasonal, and interannual variation".

\section{References}

Arcichovsky V. M. 1912, Ann. Inst. Polytech. Don Tsar. Alexis (Novotcherkassk), 1(17), 195

Arnold, L., Gillet, S., Lardière, O., Riaud, P., \& Schneider, J. 2002, A\&A, 392, 231

Arnold, L. 2008, Space Sci. Revs, 135, 323

Danjon, A. 1928, Ann. Obs. Strasbourg, 2, 165

Fossat E. 2011, arXiv:1101.3210

Hamdani, S., Arnold, L., Foellmi, C., Berthier, J., Billeres, M., Briot, D., François, P., Riaud, P., \& Schneider, J. 2006, A\&SA, 460, 617

Jacquemoud, S. \& Barret, F.1990, Remote Sensing of Environment, 34, 75

Kiang, N. Y. 2008, Scientific American, 298, 12

Tikhoff, G. A. 1914, Mitteilungen der Nikolai-Hauptsternwarte zu Poulkovo, $n^{\circ} 62$, Band $V I_{2}$, 15

Verhoef, W. 1984, Remote Sensing of Environment, 16, 125

Woolf, N. J., Smith, P. S., Traub, W. A., \& Jucks, K. W. 2002, ApJ, 99, 225 